\title{
Discovering Where We Excel: How Inclusive Turn-Taking in Conversation Improves Team Performance
}

\author{
Ki-Won Haan \\ Tepper School of Business, Carnegie \\ Mellon University, Pittsburgh, PA, \\ USA \\ khaan@andrew.cmu.edu
}

\author{
Christoph Riedl \\ D'Amore-McKim School of Business, \\ Northeastern University, Boston, MA, \\ USA \\ c.riedl@neu.edu
}

\author{
Anita Williams Woolley \\ Tepper School of Business, Carnegie \\ Mellon University, Pittsburgh, PA, \\ USA \\ awoolley@andrew.cmu.edu
}

\begin{abstract}
In this paper, we examined how inclusive turn-taking in team conversation improves performance. Inclusive turn-taking is defined as a collective speaking pattern where different team members speak in succession. This stands in contrast to exclusive turn-taking, where individual members monopolize the speaking turns. We developed an algorithm to measure inclusive turn-taking in team dialogue. We theorized and tested the indirect effects of team inclusive turn-taking on performance via team skill use, and the moderation effects of team task strategy using a sample of 150 participants randomly assigned to three-person teams.
\end{abstract}

\section{CCS CONCEPTS}

- Human-centered computing $\rightarrow$ Collaborative and social computing; Collaborative and social computing theory, concepts and paradigms; • Applied computing $\rightarrow$ Law, social and behavioral sciences; Psychology.

\section{KEYWORDS}

Team communication, Team process, Sequence analysis, Collaborative problem solving

\section{ACM Reference Format:}

Ki-Won Haan, Christoph Riedl, and Anita Williams Woolley. 2021. Discovering Where We Excel: How Inclusive Turn-Taking in Conversation Improves Team Performance. In Companion Publication of the 2021 International Conference on Multimodal Interaction (ICMI '21 Companion), October 18-22, 2021, Montréal, QC, Canada. ACM, New York, NY, USA, 6 pages. https://doi.org/10.1145/3461615.3485417

\section{INTRODUCTION}

Individuals and organizations often look to teamwork to address their most difficult problems. Teams offer the possibility of accomplishing goals that individuals cannot accomplish on their own by achieving synergy in the combination of skills and effort of individual members. Communication is the main vehicle for accomplishing the coordination needed to make synergy possible. Teams need to share their unique knowledge and skills, and jointly integrate these

Permission to make digital or hard copies of all or part of this work for personal or classroom use is granted without fee provided that copies are not made or distributed for profit or commercial advantage and that copies bear this notice and the full citation on the first page. Copyrights for components of this work owned by others than ACM must be honored. Abstracting with credit is permitted. To copy otherwise, or republish, to post on servers or to redistribute to lists, requires prior specific permission and/or a fee. Request permissions from permissions@acm.org.

ICMI '21 Companion, October 18-22, 2021, Montréal, QC, Canada

(c) 2021 Association for Computing Machinery.

ACM ISBN 978-1-4503-8471-1/21/10 . \$ \$15.00

https://doi.org/10.1145/3461615.3485417 inputs for collaborative problem solving [1,2]. The improved skill use of team members and better coordination of task strategy are thought to be among the products of equal contribution to conversation in groups, which extant work demonstrates is a predictor of collective intelligence $[3,4]$.

In this paper, we explore the patterns of team member contribution to conversation in deeper detail to understand how and why patterns of equal contribution to conversations are beneficial. It may be the case, for example, that not all patterns of equal contribution are helpful. For instance, by most commonly-used metrics, a group could exhibit equal contribution to conversation even if one person does all of the talking for one portion of the interaction, followed in sequence by other members dominating the conversation. But we theorize that the involvement of multiple team members in successive speaking turns is an important aspect of truly productive team conversation, and a key driver of the benefits that lead to synergy. To examine this, here we analyze the effects of more specific, inclusive speaking patterns rather than more global measures of distribution of speaking time or turns.

Building on extant work on collective intelligence [5], we theorize that inclusive turn-taking, which is a collective speaking pattern where different team members speak in succession, can enhance team performance by improving team collaboration processes. Specifically, we anticipate that the consistent engagement of all members enabled by inclusive turn-taking will enhance team member skill use, which we expect will mediate the effect of inclusive turn-taking on performance. Another important input will be the quality of the team's task strategy, as teams that manage to coordinate member inputs to insure that work gets accomplished will also reap the most benefit from using members' skills [6]. Figure 1 depicts the conceptual model. To test these predictions, we examined the speech and process data of 150 participants working in 50 teams. We conducted sequence analysis [7] on teams' conversational turn-taking to develop a measure of inclusive turn-taking in teams, and examined its relationship with team skill use and task strategy.

\section{THEORY DEVELOPMENT}

We theorize that inclusive turn-taking in team conversationdefined as a turn-taking pattern in conversation in which different team members speak in successive turns-reflects an open environment where members feel psychologically safe to share their ideas and thoughts as well as listen to others. Consequently, teams exhibiting such conversational patterns are more likely to discover and use members' skills. If teams also coordinate effective task strategy, they will achieve even more benefit from members' skills, 


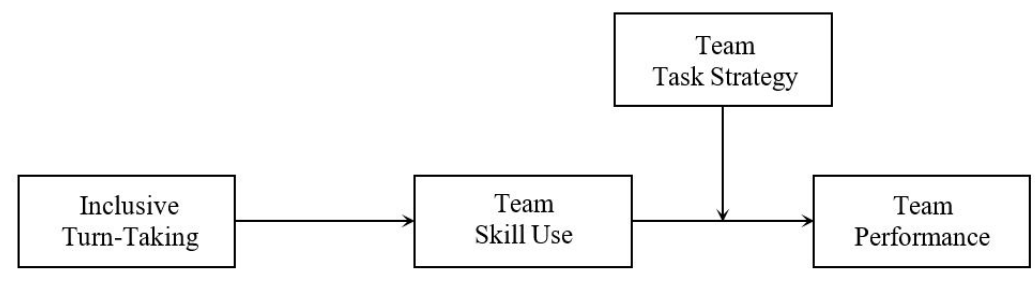

Figure 1: Theorized model

as the team will accomplish a larger volume of work and this will be reflected in even better performance. Here we draw on work on inclusive climate [8], knowledge specialization [9], and collaborative and open communication in teams [10] to theorize and test the implications of team inclusive turn-taking on team performance.

\subsection{Inclusive turn-taking and the mediating role of team skill use}

Discussion in teams can allow members to demonstrate who is good at what, facilitating the identification of member expertise [11]. In particular, researchers have found that frequency and amount of task-oriented communication in the early phase of team development predicted expertise location and cognition-based trust [12]. In an extension of this approach, we suggest that team members can figure out faster who has expertise in a particular knowledge domain if their communication involves inclusive turn-taking.

Specifically, as more members participate in the discussion more regularly, inclusive turn-taking is likely to surface more ideas, information, and facilitate integration of diverse perspectives in a collaborative manner. This results in a more consistent level of engagement of all members, and provides the team with more opportunities to become familiar with the knowledge of each member. Doing so also provides the team with more opportunities to build on each other's knowledge to generate synergy by actively integrating the different perspectives [8].

We theorize that an inclusive turn-taking pattern in conversation reflects a psychologically safe environment where members will feel more comfortable talking openly with others [13] and consequently can demonstrate their expertise more, making their knowledge more accessible, such that they can more precisely determine who is well-versed in which knowledge domain. Consequently, inclusive turn-taking allows for members to be more aware of where expertise and skills reside. This improved shared knowledge of member expertise is, in turn, likely to enable appropriate use of differentiated team member skills. That is, team members will be better able to step forward and/or defer to fellow members to "take the lead" when a task demands their unique knowledge or ability. The result is better team skill use, as reflected in specialized effort in teams, where member effort is allocated to tasks for which their particular knowledge and skills are highly applicable. By contrast, in teams where members are unequally engaged in communication, either during certain periods or throughout their work, the team is less likely to discover each other's specialized knowledge or skills nor feel empowered to step forward to declare it when appropriate.
In this regard, we expect such teams will exhibit poor skill use, reflected in less specialized effort, where member work contributions will have a very weak relationship with their relative knowledge and skill on different tasks.

Hypothesis 1: Team inclusive turn-taking will be positively associated with team skill use.

Team skill use will improve team performance by facilitating efficient division of cognitive labor and matching problems with the most skilled member $[14,15]$. Specifically, when team members accurately recognize team member expertise, they are better equipped to make the most use of each member by delegating effort, such that on a given task, the most skilled member contributes the most while the least skilled member contributes the least to problem solving. Importantly, such a high congruence of team member skill and effort will allow for a team to align member expertise with participation and influence, thereby generating better solutions. That is, effective team skill use is a reflection of team members negotiating their respective roles in a way that most skilled members are encouraged to participate and contribute more, and have a greater influence on determining appropriate actions for given tasks. Consistent with this argument, Mayo and colleagues [16] found that the alignment of member expertise and participation is associated with the alignment of member expertise and influence, which in turn predicted improved team decision-making performance. Taken together, we predict the following:

Hypothesis 2: Team skill use will be positively associated with team performance.

Hypothesis 3: Team inclusive turn-taking will have an indirect effect on team performance through skill use.

\subsection{The moderating role of team task strategy}

While team skill use is an important process for teams to perform at the highest level possible, another important consideration is the coordination of team task strategy [6]. Indeed, extant work demonstrates that teams of experts can underperform non-expert teams when they fail to coordinate and integrate their inputs appropriately [17]. For example, some teams may use expertise by delegating all of the work in an expertise domain to the most expert member while the rest of the team does not contribute. This can occur when members other than the most skilled member feel demotivated and disengaged, or fear making a mistake in front of others who are more capable in a particular area. Consequently, while the team may be making good use of expertise, they would also be underutilizing the rest of their resources to get all of the work accomplished. This will typically manifest in areas of work 
left unfinished, as often the expert member cannot both solve the more difficult expertise-relevant problems and finish all of the other work without the help of the team.

On the other hand, in a well-coordinated team, members will be better able to strategically regulate collective goal striving and attend together to the task at hand, such that they can cover more required elements of each task. Non-expert members could handle parts of a task where expertise is less essential, or else assist expert members in other ways. Therefore, we posit that team skill use, as reflected in member specialized effort, will have a stronger positive relationship with team performance when the team also exhibits stronger coordination in task strategy, manifested in less unfinished work or higher task completion rates.

Hypothesis 4a: Team task strategy will moderate the effects of skill use on team performance, such that the positive relationship is stronger when the task completion rate is higher.

Hypothesis 4b: Team task strategy will moderate the indirect effect of team inclusive turn-taking on team performance through skill use. Specifically, the indirect effect will be stronger when teams also exhibit strong task strategy compared to average or weak task strategy.

\section{METHOD}

\subsection{Participants and procedures}

We recruited 150 participants through the subject pool of a university in the northeastern US (mean age $=22.77$, sd $=4.01$; female $=50 \%$ ). Participants were randomly assigned to 50 three-person teams. Participants completed a battery of problem-solving tasks, administered through the Platform for Group Studies (POGS) [3, 18]. The platform provides task instructions, a tutorial, and a shared workspace for team members to work synchronously and see the work of team members throughout their collaboration. Each team member worked on a separate laptop, seated face-to-face around a table with teammates, and they wore a headset during the interaction to capture their individual speaking behavior. POGS captures a detailed log of each member's keystrokes across all tasks which enables us to construct detailed metrics of team collaboration process.

\subsection{Measures}

3.2.1 Team inclusive turn-taking. To measure team inclusive turntaking, we first took each individual members' audio data, converting each individual member's utterances into a binary data stream ( $0=$ no speech activity; $1=$ speech activity) using a 30 millisecond sampling rate via WebRTC Voice Activity Detector (VAD). We then superimposed the data streams of all three members to identify speaking turns. All contiguous sequences of a particular member speaking were considered part of one speaking turn; when a second person spoke, that was coded as the beginning of a new speaking turn. Periods of silence were excluded; however, when one person's speaking turn was followed by a period of silence and then the same person spoke again, that was coded as two consecutive speaking turns by the same person. In determining a unit of analysis for analyzing speaking turn sequences, we decided that sequences of three speaking turns constituted a theoretically-meaningful unit of analysis, as teams consisted of three members in this study, and within a set of three speaking turns we can examine how many of the three team members contributed. Thus, we programmed the algorithm to label each three-turn sequence based on how many different members spoke within it. Team inclusive turn-taking was operationalized as the number of speech segments or units where all three team members spoke, irrespective of the order in which they spoke-that is, a sequence in which member A spoke, then $\mathrm{B}$, then $\mathrm{C}$ is treated the same as another that started with member $\mathrm{C}$, then $\mathrm{B}$, then $\mathrm{A}$, and all other permutations including all three members in the sequence.

3.2.2 Team skill use. To gauge the degree to which a team made use of member skills, we analyzed both the level of skill each member demonstrated for each of the tasks, and also how much relative effort they put into each task. To evaluate individual member skills, for each task we measured the number of accurate or correct responses each member contributed as a percentage of their total responses attempted. We examined the amount of effort each member contributed to each task by counting the number of keystrokes each participant made as a percentage of the team's total keystrokes for the task, consistent with measures of effort in existing studies [5]. Then, to evaluate team skill use, we calculated the congruence of member skill level and amount of effort for each task, and used the average of skill congruence coefficients across tasks to operationalize team skill use for each team.

3.2.3 Team task strategy. Team task strategy provides a measure of the quality of the team's coordination of members' work by capturing the degree to which the team completed all parts of each task versus the leaving tasks incomplete [5]. This reflects team task strategy, as it differentiates teams that choose to divide the work when possible to cover everything from those that work together more to achieve high accuracy or quality on a portion of the work. For team task strategy, we first computed the percentage of work completed on each task (regardless of its quality or accuracy), and then calculated the average completion across all tasks for each team.

3.2.4 Team performance. Team performance was measured based on the weighted standardized performance score teams earned on three different sets of problems, including matrix reasoning, Sudoku puzzle, and word unscramble problems. Specifically, each set of problems was scored for accuracy, and the scores were standardized for each using the means and standard deviations from a recent meta-analysis that included the same problems [5]. Then using the weights of each task, an overall performance score for each team was calculated.

\subsection{Analysis}

To test our hypotheses, we performed path analysis using MPlus 8.3 [19]. Study variables were standardized. In testing the indirect effects, we used the bootstrapping application of Preacher and Hayes [20] to construct bias-corrected confidence intervals based on 10,000 resamples. In testing Hypothesis 3 (the indirect effect of team inclusive turn-taking on team performance via team skill use), to control for the number of inputs used to measure skill use to insure comparability between teams, we controlled for team task strategy. The interaction term of team skill use and task strategy was included 
Table 1: Descriptive statistics and intercorrelations of study variables ${ }^{\mathrm{a}}$

\begin{tabular}{lrrrrr}
\hline \multicolumn{1}{c}{ Variable } & Mean & SD & 1 & 2 & 3 \\
\hline 1. Team inclusive turn-taking & 58.54 & 30.80 & - & & \\
2. Team task strategy & .47 & .11 & -.04 & - & \\
3. Team skill use & .75 & .24 & $.35^{*}$ & -.16 & - \\
4. Team performance & -.27 & .66 & .04 & $.68^{* * *}$ & .12 \\
\hline
\end{tabular}

${ }^{a}$ Note. $n=50 .{ }^{*} p<.05 .{ }^{* * *} p<.001$.

Table 2: Path analysis results ${ }^{\mathrm{a}}$

\begin{tabular}{|c|c|c|c|c|c|c|}
\hline \multirow{2}{*}{$\begin{array}{l}\text { Main/Interaction Effect } \\
\text { Team inclusive turn-taking }\end{array}$} & \multicolumn{2}{|c|}{ Skill Use } & \multicolumn{4}{|c|}{ Team Performance } \\
\hline & $.35^{* *}$ & $(0.13)$ & -.01 & $(0.07)$ & -.01 & $(0.07)$ \\
\hline Team task strategy & & & $.47^{* * *}$ & $(0.07)$ & $.44^{* * *}$ & $(0.06)$ \\
\hline Team skill use & & & $.16^{*}$ & $(0.07)$ & $.17^{* *}$ & $(0.07)$ \\
\hline Team skill use $\times$ task strategy & & & & & $.16^{*}$ & $(0.07)$ \\
\hline$R^{2}$ & .12 & & .54 & & .59 & \\
\hline Indirect Effect & & & Estimate & \multicolumn{3}{|c|}{ 95\% Bias-corrected CI } \\
\hline Team inclusive turn-taking $\rightarrow$ & ill use) & & .05 & \multicolumn{3}{|c|}{$[.012, .132]$} \\
\hline
\end{tabular}

${ }^{\mathrm{a}}$ Note. $n=50$. Standard errors in parentheses. ${ }^{*} p<.05 .{ }^{* *} p<.01 .{ }^{* * *} p<.001$.

in the model to test the moderation predicted in Hypothesis 4a and the conditional indirect effect predicted in Hypothesis $4 \mathrm{~b}$.

\section{RESULTS}

Descriptive statistics and intercorrelations for the study variables are reported in Table 1 . The results of path analysis and indirect effects are reported in Tables 2 and 3

Hypothesis 1 predicted that inclusive turn-taking in teams will be associated with skill use, or the level of agreement between relative member skills and effort on each task. The result shown in Table 2 indicates that team inclusive turn-taking was positively associated with team skill use ( $b=.35, p<.01)$, lending support to Hypothesis 1. Hypothesis 2 predicted a positive relationship between team skill use and team performance, controlling for team task strategy. The effect of team skill use on performance was positive and significant $(b=.16, p<.05)$. In addition, Hypothesis 3 predicted that there would be an indirect effect of team inclusive turn-taking on performance via skill use; the results of the indirect effects analysis were consistent with this prediction and the confidence interval excludes zero (indirect effect $=.05,95 \%$ bias-corrected $\mathrm{CI}=.012$, $.132 ; 99 \%$ bias-corrected CI $=.002, .161$ ).

Hypothesis 4a predicted that team task strategy will moderate the effects of skill use on team performance, such that the positive relationship is stronger when the task completion rate is higher. The statistical interaction between team skill use and task strategy is significant $(b=.16, p<.05$; see Table 2$)$. The interaction is graphically depicted in Figure 2. Simple slopes analysis at one standard deviation above and below the sample mean [21] reveals that when team strategy was highly coordinated, such that team members completed all or most of all tasks, the relationship between team skill use and performance is significant (simple slope $=.34, t=3.60$, $p<.001$ ), but not when team task strategy was weak (simple slope $=.01, t=.14, p=n s$ ).

Last, with respect to Hypothesis $4 \mathrm{~b}$, we examined the conditional indirect effect of team inclusive turn-taking on performance via skill use at high (1 standard deviation above the mean), moderate (mean), or low (1 standard deviation below the mean) levels of task strategy (see Table 3). Significant conditional indirect effects were observed when levels of task strategy were moderate (indirect effect $=.06 ; 95 \%$ bias-corrected $\mathrm{CI}=.014, .136$ ) to high (indirect effect $=.12,95 \%$ bias-corrected CI $=.014, .284$ ). Across all levels, however, the $95 \%$ confidence interval of the index of moderated mediation included zero (index $=.06,95 \%$ bias-corrected bootstrap $\mathrm{CI}=-.011, .153)$. We further explored the conditional effects of task strategy at more finely-specified levels with the Johnson-Neyman technique. For teams who scored above the 38th percentile on task strategy, the strength of their task strategy significantly moderated the indirect effect of inclusive turn-taking on performance via skill use. In other words, inclusive turn-taking was particularly helpful to teams in translating their use of member skill into performance if they were also performing at a moderate-to-high level with respect to task strategy. Thus Hypothesis $4 \mathrm{~b}$ was partially supported.

\section{CONCLUSIONS AND DISCUSSION}

Extant research has demonstrated the importance of a high level and relative equality of contribution to communication for collective intelligence. In this study, we dig more deeply into the patterns of communication that drive potential benefits. In doing so, we developed a new construct and measure to capture team inclusive turn-taking, based on a sequence analysis, and theorized and tested the mechanism of inclusive turn-taking. In particular, we focused on the mediating role of a team's appropriate use of team member skills and the moderating role of a team's task performance strategy. 


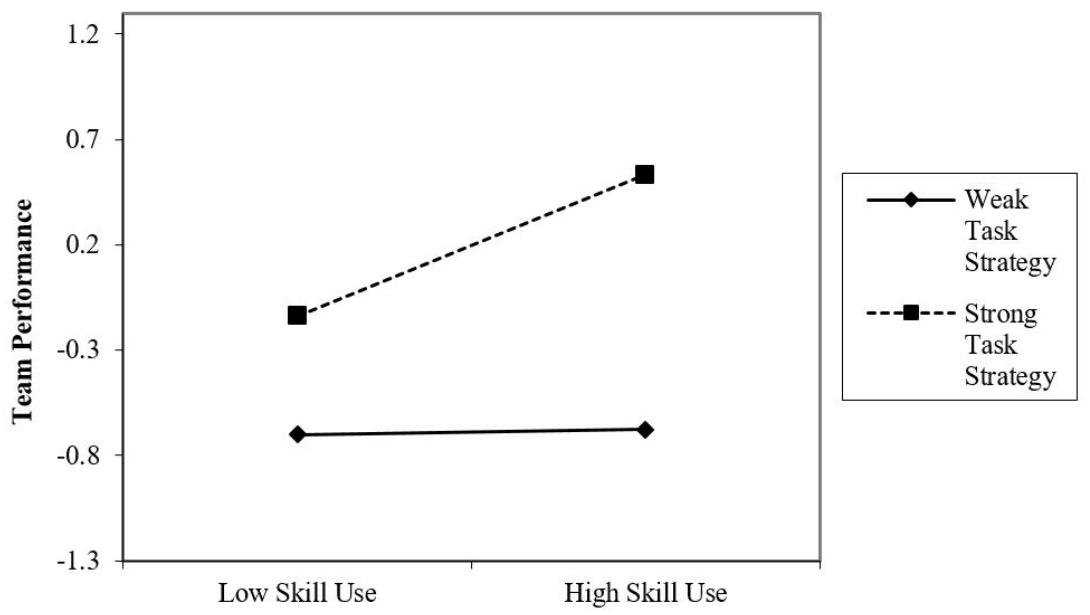

Figure 2: Moderation effects of team task strategy on the relationship between skill use and performance

Table 3: Conditional indirect effects at high, mean, low levels of team task strategy for inclusive turn-taking

\begin{tabular}{|c|c|c|c|c|c|}
\hline Independent variable & Mediator & Moderator & Indirect effects & $\begin{array}{c}\text { LL } 95 \% \text { BC } \\
\text { bootstrap CI }\end{array}$ & $\begin{array}{c}\text { UL } 95 \% \text { BC } \\
\text { bootstrap CI }\end{array}$ \\
\hline \multirow{3}{*}{ Inclusive turn-taking } & \multirow{3}{*}{ Skill use } & Strong task strategy & .116 & .014 & .284 \\
\hline & & Moderate task strategy & .060 & .014 & .136 \\
\hline & & Weak task strategy & .004 & -.079 & .061 \\
\hline
\end{tabular}

We found that inclusive turn-taking is positively related to team skill use, and when this is combined with strong task strategy, it enables higher team performance.

While this research highlights a number of new insights about group communication and collaboration, there are also many different directions this work suggests for future research. For instance, an important question relates to the conditions that lead groups to develop inclusive turn-taking patterns. We theorize a role for group climate and psychological safety, although an important unanswered question concerns which of these comes first? Do psychologically-safe groups engage in inclusive turn-taking in their communication, or does this communication pattern create psychological safety? Future work could look for settings in which these variables could be experimentally manipulated in a meaningful way to assess this kind of question. In addition, there are likely a host of variables related to team composition and group member relationships which play an important role and should be evaluated in future research as well.

This research represents an important contribution to research on multimodal human interaction, and ultimately human-computer interaction. This study builds on traditional research on group process by creating machine-based measures of communication and team collaboration processes that capture the quality of group interaction and predict performance. Such measures lay the groundwork for the eventual involvement of machine-based tools and interventions to help groups whose communication and collaboration patterns diverge from those we have shown to be productive. Consequently, this work provides both a conceptual framework and methodological pathway for both improving human collaboration and enabling artificial social intelligence for enhancing teamwork.

\section{ACKNOWLEDGMENTS}

This research was sponsored by the Army Research Office under Grant Number W911NF-16-1-0005 and the US Army Research Lab under Grant Number W911NF-19-2-0135.

\section{REFERENCES}

[1] James Larson JR. 2013. In search of synergy in small group performance. New York, NY: Psychology Press

[2] Jean Macmillan, Elliot E. Entin, and Daniel Serfaty. 2004. A framework for understanding the relationship between team structure and the communication necessary for effective team cognition. In E. Salas, S.M. Fiore, \& J. Cannon-Bowers, (Eds.), Team cognition: Process and performance at the inter-and intra-individual level. Washington, DC: APA.

[3] David Engel, Anita Williams Woolley, Lisa X. Jing, Christorpher F. Chabris, and Thomas W. Malone. 2014. Reading the mind in the eyes or reading between the lines? Theory of mind predicts collective intelligence equally well online and face-to-face. PloS ONE, 9(12), Article e115212.

[4] Anita Williams Woolley, Christopher F. Chabris, Alex Pentland, Nada Hashmi, and Thomas W. Malone. 2010. Evidence for a collective intelligence factor in the performance of human groups. Science, 330(6004), 686-688.

[5] Christoph Riedl, Young Ji Kim, Pranav Gupta, Thomas W. Malone, and Anita Williams Woolley. 2021. Quantifying collective intelligence in human groups. Proceedings of the National Academy of Sciences, 118(21).

[6] Hackman, J. R. (1987). The design of work teams. In J. W. Lorsch (Ed.), Handbook of organizational behavior (pp. 315-342). Prentice Hall.

[7] Benjamin Cornwell. 2015. Social sequence analysis: Methods and applications. Cambridge, UK: Cambridge University Press. 
[8] Lisa H. Nishii. 2012. The benefits of climate for inclusion for gender-diverse groups. Academy of Management fournal, 56(6), 1754-1774.

[9] Yuqing Ren and Linda Argote. 2011. Transactive memory systems 1985-2010 An integrative framework of key dimensions, antecedents, and consequences. Academy of Management Annals, 5(1), 118-229.

[10] Charles A. O'Reilly III and Karlene H. Roberts. 1977. Task group structure, communication, and effectiveness in three organizations. Fournal of Applied Psychology, 62(6), 674-681.

[11] Kyle Lewis. 2004. Knowledge and performance in knowledge-worker teams: A longitudinal study of transactive memory systems. Management Science, 50(11) 1519-1533.

[12] Prasert Kanawattanachai and Youngjin Yoo. 2007. The impact of knowledge coordination on virtual team performance over time. MIS Quarterly, 783-808.

[13] Amy Edmondson. 1999. Psychological safety and learning behavior in work teams. Administrative Science Quarterly, 44(2), 350-383.

[14] Richard L. Moreland. 1999. Transactive memory: Learning who knows what in work groups and organizations. In L. Thompson, D. Messick, \& J. Levine (Eds.), Shared cognition in organizations: The management of knowledge (pp. 3-31). Mahwah, NJ: Erlbaum.
[15] Daniel M. Wegner. 1995. A computer network model of human transactive memory. Social Cognition, 13(3), 319-339.

[16] Anna T. Mayo, Anita Williams Woolley, and Rosalind M. Chow. 2020. Unpacking participation and influence: diversity's countervailing effects on expertise use in groups. Academy of Management Discoveries, 6(2), 300-319.

[17] Woolley, A. W., Gerbasi, M. E., Chabris, C. F., Kosslyn, S. M., \& Hackman, J. R. (2008). Bringing in the experts: How team composition and work strategy jointly shape analytic effectiveness. Small Group Research, 39(3), 352-371.

[18] Young Ji Kim, David Engel, Anita Williams Woolley, Yu-Ting Jeffrey Lin, Naomi McArthur, and Thomas W. Malone. 2017. "What Makes a Strong Team?: Using Collective Intelligence to Predict Team Performance in League of Legends." in 20th ACM Conference for Conference on Computer-Supported Cooperative Work and Social Computing CSCW'17. Portland, OR.

[19] Bengt O. Muthén and Linda K. Muthén. 2017. Mplus user's guide (8th ed.). Los Angeles, CA: Muthén \& Muthén.

[20] Kristopher J. Preacher and Andrew F. Hayes. 2008. Asymptotic and resampling strategies for assessing and comparing indirect effects in multiple mediator models. Behavior Research Methods, 40, 879-891.

[21] Aiken, L. S., \& West, S. G. (1991). Multiple regression: Testing and interpreting interactions. Newbury Park, CA: Sage. 